\title{
Consumption of a Smoothie or Cereal-Based Breakfast: Impact on Thirst, Hunger, Appetite and Subsequent Dietary Intake
}

Courtney Myers ${ }^{1}$, Danielle McCartney ${ }^{3}$, Ben Desbrow ${ }^{1}$, Saman Khalesi ${ }^{4}$, Christopher Irwin ${ }^{1,2^{*}}$

${ }^{I}$ School of Allied Health Sciences, Griffith University, Gold Coast, Australia

${ }^{2}$ Menzies Health Institute Queensland, Gold Coast, Australia

${ }^{3}$ The University of Sydney, Faculty of Science, School of Psychology, Sydney, New South Wales, Australia

${ }^{4}$ Central Queensland University, Appleton Institute and School of Health, Medical and applied Sciences, Brisbane, Queensland, Australia

\section{Corresponding Author:}

Dr Chris Irwin

School of Allied Health Sciences, Griffith University

Parklands Drive, Southport QLD, Australia 4222.

Email: c.irwin@griffith.edu.au

Phone: +61 756787344 


\begin{abstract}
:
Smoothies are a popular breakfast option. However, liquids may evoke weaker satiation than nutritionally-comparable semi-solid and solid foods. This study examined consumption of Cereal \& Milk (CM) or a nutritionally comparable Fruit Smoothie (FS) for breakfast on subsequent dietary behaviours, in a controlled laboratory setting. Twenty-five participants (age $25 \pm 6 \mathrm{y}$ ) completed 3 trials, receiving either CM or FS for breakfast. Afterwards, participants remained isolated for $4 \mathrm{~h}$ with ad libitum access to foods/beverages. A repeat trial (CM or FS) allowed exploration of normal variability. Post-breakfast energy intake (EI) (CM=1465(2436) vs $\mathrm{FS}=1787(3190) \mathrm{kJ}$, Median(IQR), $p=0.099)$, time to intake of next food/fluid (meal latency) $(\mathrm{CM}=146(97)$ vs $\mathrm{FS}=180(100) \mathrm{min}, p=0.127)$, and subjective hunger, desire to eat, fullness and thirst ratings were similar between conditions $\left(p s^{\prime}>0.05\right)$. The mean coefficient of variation for EI and meal latency were $41 \%$ and $21 \%$, respectively. Consumption of a FS does not negatively impact acute EI and meal latency.
\end{abstract}

Keywords: smoothie; breakfast; liquid; solid; food form; subsequent dietary intake 


\section{Introduction:}

Breakfast is often described as the most important meal of the day (Spence, 2017). Indeed, recommendations from many professional nutrition authorities suggests that regularly eating breakfast is associated with a range of positive outcomes, including better overall diet quality and maintenance of a healthy body weight (Academy of Nutrition and Dietetics, 2014, British Dietetic Association, 2016, Food Safety Authority of Ireland, 2011, National Health and Medical Research Council, 2013, New Zealand Ministry of Health, 2012, Public Health England, 2016). However, this has been challenged in a recent systematic review and meta-analysis, with suggestions that eating breakfast results in higher total daily energy intakes and therefore may inhibit attempts to lose weight (Sievert et al., 2019). To more thoroughly understand the influence of breakfast consumption (or not) on overall dietary behaviour and energy intake, clearly, some consideration of different breakfast options is also required.

The influence of manipulating the type of breakfast consumed on appetite control and subsequent dietary behaviour has received due scientific attention (Berti et al., 2015, Fallaize et al., 2013, Rosi et al., 2018). For example, Berti et al. (2015) found that consuming a fruit-salad with a standard breakfast (rice and whole wheat flakes + milk) induced greater satiety sensations compared to consuming fruit juice with the standard breakfast or the standard breakfast alone. Similarly, Fallaize et al (2013) observed increased satiety, less hunger, a lower desire to eat, and lower intake of energy at subsequent meals after participants consumed a breakfast containing eggs compared to both cereal- and croissant-based meals. In contrast, a recent study demonstrated no differences in the amount of food ingested at a subsequent meal (i.e. lunch) after consuming three different breakfast meals (with minor nutritional variances) or a fasting control (Rosi et al., 2018). Nonetheless, breakfast type may be an important determinant of energy intake and diet 
quality due to its effects on satiety-related sensations (thus, appetite control) at subsequent meal times (Berti et al., 2015, Fallaize et al., 2013) or impact on postprandial metabolic profiles (Rosi et al., 2018).

While dietary behaviours are becoming less predictable as a result of dieting trends and busy lifestyle schedules that alter normal eating patterns (Davis et al., 2016, Presser and Ward, 2011, Zizza et al., 2001), breakfast still appears to be a commonly consumed meal (Haines et al., 1996, Kant and Graubard, 2006, Williams, 2005). Indeed, three quarters of adults within western cultures such as Australia (Williams, 2005) and the United States (Haines et al., 1996, Kant and Graubard, 2006) indicate that they consume breakfast on most days of the week. However, recent data indicate a change in the type of breakfast foods being consumed, with individuals indicating a preference for liquid-based meals (e.g. fruit smoothies) over traditional (i.e. cereal-based and cooked meals) breakfast options (The Neilson Company, 2014). Indeed, a recent Australian survey (exploring attitudes, beliefs and behaviours on consumption of smoothies) of 833 adults (79\% female) revealed that $94 \%$ of the sample population consumed smoothies, with $\sim 39 \%$ doing so for breakfast in lieu of other foods (McCartney et al., 2018). While smoothies may provide an efficient means to increase intakes of core food groups (e.g. fruit, vegetables, dairy and/or alternatives) (Bates and Price, 2015), some evidence suggests that caloric liquids have weaker satiating effects (and may therefore, promote greater subsequent energy intake) compared to nutritionallycomparable semi-solid and solid foods (Almiron-Roig et al., 2013, Mourao et al., 2007).

A recent pilot investigation comparing the impact of consuming a Fruit Smoothie (FS) versus a nutritionally-equivalent Cereal \& Milk (CM) breakfast for three consecutive days, found that the type of breakfast had negligible influence on subsequent dietary behaviours (e.g. $324 \mathrm{~kJ}$ difference in mean daily energy intake) (McCartney et al., 2019). However, dietary behaviour following 
breakfast (for the remainder of the day) occurred within a free-living environment, with data collected via self-reported food diaries. This may have increased the risk of participants altering their normal eating behaviours (as they are aware of it being monitored) and/or inaccurate reporting (Ortega et al., 2015).

Therefore, the aim of the current study was to determine the impact of consuming a FS or a nutritionally-equivalent $\mathrm{CM}$ breakfast on subsequent acute (4 h post-breakfast) dietary behaviour in a controlled laboratory setting. It was hypothesised that consuming a FS for breakfast would result in shorter meal latency (i.e. time to seek next calorie-containing food/beverage), higher subsequent energy intake, and lower subjective appetite ratings (i.e. greater hunger and desire to eat, and lower fullness and thirst) compared to CM.

\section{Materials and Methods:}

\section{Participants:}

Twenty-five healthy participants ( $n=11$ male, 14 female; age: $25 \pm 6$ y; BMI: $22.7 \pm 2.3 \mathrm{~kg} \cdot \mathrm{m}^{-2}$; values are Mean \pm Standard Deviation $[\mathrm{SD}])$ took part in this investigation. Sample size was determined using power calculation software ( $\mathrm{G}^{*}$ Power Version 3.1.9.2, University of Kiel, Germany, 2014) and consideration for attrition ( 10\%). Employing a Cohen's $d_{z}$ effect size of 0.6, based on data from Flood-Obbagy \& Rolls (2009) who identified a significant difference in ad libitum energy intake at a meal following consumption of different food forms in a pre-load meal (i.e. apple juice with fibre vs. intact apple pieces), a power $(1-\beta)=0.8$ and $\alpha=0.05,24$ participants were required to detect a significant difference in post-breakfast energy intake between experimental treatments. Participants were informed of the nature and possible risks of the study before providing written informed consent. However, participants were unaware that some 
parameters (i.e. time to next eating/drinking occasion following breakfast) were being monitored. They were instead told that the purpose of the study was to investigate the influence of different breakfasts on cognitive function (sham tasks were administered to maintain this deception). Each participant was given the opportunity to withdraw their data once data collection activities for the entire study were complete. The investigation was approved by the Griffith University Human Research Ethics Committee (Ref No: 2019/045), and the procedures were conducted in accordance with the principles outlined by the agreement of Helsinki.

\section{Study Design:}

This study employed a within-subject, randomised and controlled design. The experimental protocol is displayed in Figure 1. All participants completed three trials, with a minimum of three days between trials. During each trial participants received one of two different isocaloric breakfasts: (1) Cereal \& Milk (CM) or (2) Fruit Smoothie (FS), then were given access to a variety of foods/beverages, which they could consume ad libitum over a $4 \mathrm{~h}$ period. In the first trial, $n=12$ participants received CM and $n=13$ received FS as the breakfast meal. This trial was used as familiarisation to expose participants to the foods/beverages in the ad libitum eating environment, but also to determine variability (coefficient of variation, CV) in subsequent dietary intake with repeated trials. Breakfast treatments for the two subsequent experimental trials were randomised and counterbalanced for order using an incomplete Latin-square design. Subjective ratings of hunger, thirst, desire to eat and fullness were recorded throughout trials (immediately before breakfast, immediately after breakfast, and at 20, 40, 60, 120, 180 and 240 min after breakfast). Dietary intake during the $4 \mathrm{~h}$ post-breakfast period and the time to first consumption of energycontaining foods/beverages during this period were recorded covertly. 


\section{Preliminary Screening:}

Volunteers presented to the laboratory for initial screening at least $24 \mathrm{~h}$ prior to the first (familiarisation) trial. To confirm eligibility, individuals were required to complete a general medical history questionnaire; those reporting any medical condition or comorbidity with potential to influence outcomes of the study (e.g. diabetes) were ineligible to participate. Usual morning waking time and breakfast consumption time were then documented, along with a description of their typical "at-home" breakfast. The energy and macronutrient content of this breakfast was calculated using Foodworks ${ }^{\circledR}$ Version 8 dietary analysis software (Xyris Software Pty Ltd, Spring Hill, Australia). Only "habitual breakfast consumers" (i.e. $\geq 500 \mathrm{~kJ}$ within $2 \mathrm{~h}$ of waking $\geq 4 \mathrm{~d} \cdot$ week $^{-}$

${ }^{1}$ (McCartney et al., 2019)), were eligible to participate. Individuals were then asked to rate the acceptability of food items (those being provided in the $4 \mathrm{~h}$ post-breakfast period) on a scale of 1 (dislike) to 10 (like) (Table 1) via a Food Acceptability Questionnaire (McCartney et al., 2019) before completing the 26-Item Eating Attitudes Test (EAT-26) (Constain et al., 2014) and Three Factor Eating Questionnaire (TFEQ-R18) (de Lauzon et al., 2004) to provide information on cognitive restraint, uncontrolled eating and emotional eating behaviours; then their usual smoothie and morning tea consumption patterns were documented. Following this, participants' BMI was calculated from weight and height measurements taken with digital scales (HW-PW200; A\&D Company Ltd, Tokyo, Japan) and a stadiometer (SECA 217, ECOMED Trading Pty Ltd). Body composition (i.e. body fat percentage $[\mathrm{BF} \%]$ and fat free mass $[\mathrm{FFM}]$ ) was then measured using bioelectrical impedance analysis (BIA) (Body Composition Analyzer, SECA mBCA 525, ECOMED Trading Pty Ltd). To improve the accuracy of BIA, standardisation procedures were employed with participants asked to refrain from consuming food (3-4 h), undertaking physical activity (3-4 h), and consuming fluid (2 h) prior to the preliminary screening visit (Dehghan and 
Merchant, 2008). Urine specific gravity (USG) was also measured using a digital refractometer (USG; Pen Refractometer, ATAGO, USA) to verify euhydration (USG <1.024) prior to BIA. Exclusion criteria for the study included: (1) rating the acceptability of more than $12(50 \%)$ items on the post-breakfast list of foods as $\leq 5$; (2) self-reporting an allergy, intolerance or sensitivity to the food items or the breakfast meals; (3) exhibiting disordered eating behaviour based on the EAT-26 (score $>20$ in Part B or marking any of the "at risk" boxes in Part C); (4) classification of being underweight $(\mathrm{BMI}<18.5)$ or obese $\left(\mathrm{BMI}>30.0 \mathrm{~kg} \cdot \mathrm{m}^{-2}\right.$ ) (World Health Organization, 2018). Overall, 26 participants were assessed for eligibility, with only one participant excluded because they did not meet the definition of a "habitual breakfast consumer".

\section{Experimental Procedures:}

Standardisation Procedures:

Participants were instructed to keep a written record of all foods/beverages consumed, as well as any exercise undertaken for the $24 \mathrm{~h}$ prior to their first trial. A copy of the record was provided to participants after their first trial and they were instructed to replicate dietary/exercise behaviours in the $24 \mathrm{~h}$ prior to all subsequent trials, but to also note any changes made if these occurred. Participants were required to avoid caffeine-containing products for $12 \mathrm{~h}$ and fast from all food and beverages for $10 \mathrm{~h}$ (i.e. overnight) before all trials. Upon waking on the morning of trials, participants were asked to consume $250 \mathrm{~mL}$ of water. Participants then attended the laboratory between 06:00-08:00 $\mathrm{h}$ to commence trials (note: participants laboratory start times were recorded at the first trial and all subsequent trials commenced at the same starting time). Standardisation conditions were verbally confirmed on arrival to the laboratory and participants indicated the time they woke from sleep that morning and the number of hours they slept last night. Given that 
hydration status can influence subjective appetite ratings (Shirreffs et al., 2004), participants then provided a urine sample to determine hydration status via urine specific gravity (USG). Participants were considered euhydrated if USG was $<1.020$ (Armstrong et al., 2010). Hypohydrated participants were provided $250 \mathrm{~mL}$ water to consume within $5 \mathrm{~min}$ and a subsequent USG was collected $\sim 20$ min later. Participants were able to proceed with the trial if a USG $<1.020$ was recorded, otherwise they were asked to reschedule. One participant required additional water on their first trial; as such, they were asked to consume an additional $250 \mathrm{~mL}$ (i.e. $500 \mathrm{~mL}$ total) water upon waking before all subsequent trials.

\section{Breakfast Composition and Eating Environment:}

The CM and FS breakfasts were identical to those described in a previous study (McCartney et al., 2019). The CM contained the following ingredients (in order of energy contribution): Hi-Bran Weet Bix ${ }^{\mathrm{TM}}$ (Sanitarium ${ }^{\circledR}$, Australia) (39.1\%), full cream milk (Woolworths Select ${ }^{\circledR}$, Australia) (31.8\%), blueberries (Creative Gourmet ${ }^{\mathrm{TM}}$, Patties Foods ${ }^{\circledR}$, Australia) (17.7\%) and yoghurt (Vaalia $^{\mathrm{TM}}$, Parmalat Australia $\left.\mathrm{Ltd}^{\circledR}\right)(11.4 \%)$. Water was served alongside the CM to match the water content of the FS. The FS contained the following ingredients (in order of energy contribution): Full cream milk (Woolworths Select ${ }^{\circledR}$, Australia) (42.4\%), banana (26.2\%), blueberries (Creative Gourmet ${ }^{\mathrm{TM}}$, Patties Foods ${ }^{\circledR}$, Australia) (14.5\%), yoghurt (Vaalia ${ }^{\mathrm{TM}}$, Parmalat Australia $\left.\operatorname{Ltd}^{\circledR}\right)(11.3 \%)$, skim-milk powder (Woolworths Select ${ }^{\circledR}$, Australia) (3.0\%), psyllium husk (Woolworths Select ${ }^{\circledR}$, Australia) $(2.5 \%)$ and water. Ingredients for the FS were blended together for $60 \mathrm{~s}$ immediately prior to serving. Both breakfasts were closely matched for carbohydrate, fat, protein, fibre, mass and water content per unit of energy and were energymatched to participants' usual daily breakfast intake. If a participants' usual "at-home" breakfast 
provided more than $2,000 \mathrm{~kJ}$, a treatment breakfast containing $2000 \mathrm{~kJ}$ was first created and the additional energy was supplemented with maltodextrin powder (PolyJoule ${ }^{\mathrm{TM}} ; 16.6 \mathrm{~kJ} \cdot \mathrm{g}^{-1}$, Nutricia Australia, Macquarie Park, Australia). This methodology was employed to avoid serving very large volumes of food and fluid (e.g. $>900 \mathrm{~g}$ of smoothie). In addition, treatment breakfasts were limited to a maximum of $3,000 \mathrm{~kJ}$, as large quantities of Polyjoule ${ }^{\mathrm{TM}}$ were found to alter the sensory properties of FS during pilot testing. Participants were asked to consume their breakfasts over 10 min, however, two participants required 20 min to consume the breakfast on their first trial and were therefore instructed to repeat this at all subsequent trials.

\section{Post-breakfast Procedures and Environment:}

The $4 \mathrm{~h}$ post-breakfast period commenced immediately after participants finished their allocated breakfast. Participants were placed into a private room (isolated from others), which contained a selection of foods/beverages (Table 1), a small refrigerator and toaster. Participants were instructed to consume as little or much of the food/beverages as they liked. The presentation and quantities of food, drink and additional items (i.e. plates, cups, cutlery, serviettes) were kept consistent across trials and between participants. The food/beverages were placed on a table that was strategically positioned behind participants in an effort to avoid mindless eating that does not accurately reflect hunger and/or desire to eat (van der Laan et al., 2011). Participants were unaware their dietary behaviour was being monitored. To ensure intake during trial was accurately measured, participants were instructed to place all rubbish and unfinished items (that they started to consume) in a bin bag provided, leave their belongings outside the room, and to refrain from taking any food out of their private room (i.e. for consumption after trial completion). Participants could bring their choice of entertainment (i.e. laptop, iPad, earphones, phone, books, etc.) and use the bathroom as 
required.

\section{Data Collection:}

Visual analogue scales (VASs) were used to measure subjective ratings of hunger, thirst, desire to eat and fullness. Participants completed these measures immediately prior to, and following consumption of the treatment breakfast, and again at 20,40,60,120, 180 and 240 min postbreakfast consumption. A VAS was also used to measure breakfast palatability (pleasantness) immediately after consumption of the test breakfast. All VAS were administered via the computerised modifiable software program, Adaptive Visual Analogue Scales (Marsh-Richard et al., 2009) on a laptop computer using a $100 \mathrm{~mm}$ line between the anchors 'Not at All' and 'Extremely'.

Sham cognitive function tasks were administered to determine the time elapsed between finishing the treatment breakfast to the next eating/drinking occasion (i.e. meal latency) during the $4 \mathrm{~h}$ period, because participants were unaware this data was being collected. Participants were asked to complete the cognitive function task (a $2 \mathrm{~min}$, 4-choice reaction time task) on their first eating/drinking occasion (excluding water) and again at the end of the $4 \mathrm{~h}$ period. The software for the cognitive task recorded the start time (and hence the time of eating/drinking). The food/beverage items were weighed before and after (i.e. once the participant had vacated) each trial to determine total energy, nutrient, water and food groups servings intakes during the $4 \mathrm{~h}$ period using the Foodworks Version 8 dietary analysis software (Xyris Software Pty Ltd, Spring Hill, Australia). Participants were provided with a debriefing form on completion of the study to inform them of the true purpose of the investigation and the actual data collected, and to provide them with an opportunity to have their data excluded upon disclosure should they desire. 


\section{Statistical Analysis:}

All statistical procedures were performed using SPSS statistical analysis software, version 25 (IBM, SPSS Inc., Chicago, USA). All measures were examined for normality using the ShapiroWilk test prior to further analysis. Planned comparisons were performed to test the specific research hypothesis that breakfast type (i.e. CM vs. FS) would influence meal latency, subsequent dietary intake and subjective ratings. Normality tests for each of the main dependent variables (energy intake, macronutrient/sodium intake, food group servings intake and meal latency) revealed this data was non-normally distributed. Therefore, statistical analyses were performed using Wilcoxon signed rank tests, with effect estimates calculated as $r=\mathrm{Z} / \sqrt{n}$ (Pallant, 2011). The current study was not sufficiently powered to compare these dietary outcomes by sex; however, average energy intake is reported for the male and female subgroups. Subjective ratings of hunger, thirst, desire to eat and fullness derived from the VAS were log transformed and subjected to a 2 (Trial; CM vs FS, Familiarisation vs Repeat trial) $\times 8$ (Time) repeated measures analysis of variance (ANOVA). Pairwise comparisons applying Bonferroni correction were performed on all significant main effects. Differences between the Familiarisation and Repeat trials were quantified as the coefficient of variation (CV). One-way repeated-measures ANOVA were performed to compare pre-trial $24 \mathrm{~h}$ dietary intakes, dinner intakes, and hydration status (USG) across the three trials (Familiarisation, CM, FS). Energy intake and meal latency in the $4 \mathrm{~h}$ post-breakfast period were also analysed for trial order effects using the non-parametric Friedman's test. The Greenhouse-Geisser correction was applied when sphericity assumptions were violated. Each of the 18 items on the TFEQ-R18 during the preliminary screening was given a score between 1 and 4 and item scores were summed into raw scores for cognitive restraint, uncontrolled eating, and emotional eating. The raw scale scores were then transformed to a $0-100$ 
scale $[(($ raw score - lowest possible raw score $) /$ possible raw score range $) \times 100]$. Statistical significance was accepted as $p<0.05$. All data is reported as mean $\pm \mathrm{SD}$ (i.e. if data was normally distributed) or Median(IQR) (i.e. for non-normally distributed data).

\section{Results:}

\section{Participant Characteristics and Pre-trial Conditions:}

Of the 25 participants that took part in this study, 14 indicated that they usually consumed $\geq 1$

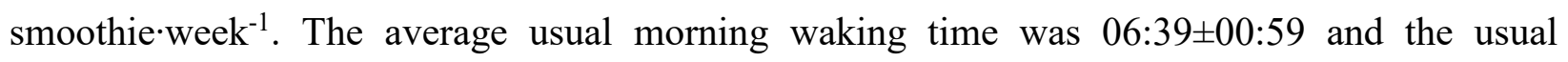
breakfast time was 07:55 $\pm 01: 11$. On average, participants consumed breakfast $6 \pm 1 \mathrm{~d} \cdot \mathrm{wk}^{-1}$ and morning tea an average of $3 \pm 3 \mathrm{~d} \cdot \mathrm{wk}^{-1}$. Data from the TFEQ-R18 indicated that none of the participants exceeded clinical thresholds for cognitive restraint, uncontrolled eating or emotional eating. During the preliminary screening, participants generally reported a liking for the food/beverage items on offer. Most (92\%) items received an average rating $\geq 8$ (out of 10) on the food acceptability questionnaire (Table 1). Only two items (Vegemite and margarine) received average ratings $<7$. All participants verbally acknowledged compliance to pre-experimental procedures upon arrival to the laboratory. Most participants awoke on the morning of each trial at the same time of day, with only subtle variations in waking time within participants. The largest difference between trials for each participant was examined, with the average difference in waking time between trials $14.4 \pm 15.3 \mathrm{~min}$ (range: $0-45 \mathrm{~min}$ ). On average, participants reported having the same amount of sleep on each trial (trial $1=6.8 \pm 1.4 \mathrm{~h}$; trial $2=6.8 \pm 1.4 \mathrm{~h}$; trial $3=6.8 \pm 1.2 \mathrm{~h}$; $p=0.880$ ). Participants commenced each trial with a similar USG (Familiarisation $=1.010 \pm 0.006$; $\mathrm{CM}=1.010 \pm 0.005 ; \mathrm{FS}=1.011 \pm 0.007 ; \mathrm{F}(2,48)=0.412, p=0.664)$. No significant differences were observed in participants' pre-trial $24 \mathrm{~h}$ dietary intakes with respect to energy $(F(2,48)=1.140$, 
$p=0.328)$, carbohydrate $(\mathrm{F}(1.45,34.82)=0.107, p=0.835)$, fat $(\mathrm{F}(1.45,34.77)=0.065, p=0.884)$, or protein $(\mathrm{F}(1.43,34.24)=1.098, p=0.326)$. Likewise, no differences were observed in participants' pre-trial dinner intakes for energy $(\mathrm{F}(1.30,31.28)=2.423, p=0.123)$, carbohydrate $(\mathrm{F}(1.07$, $25.82)=0.471, p=0.512)$, fat $(\mathrm{F}(1.29,31.14)=0.207, p=0.716)$, or protein $(\mathrm{F}(2,48)=0.803$, $p=0.454)$.

\section{Breakfast Characteristics:}

The nutritional composition of participants' usual breakfast and each of the experimental breakfasts is displayed in Table 2. The average energy content of participants' usual "at home" breakfast was estimated as 2,112 $\pm 892 \mathrm{~kJ}$ (range: $959-4012 \mathrm{~kJ}$ ). Hence, the average energy content of the experimental breakfasts was 2,006 $\pm 701 \mathrm{~kJ}$ (range: 959-3,000 kJ). All participants voluntarily consumed the entire contents of each test-breakfast. The palatability of the FS and CM breakfasts were rated as $83(20) \mathrm{mm}$ and $92(21) \mathrm{mm}$ respectively, with no significant difference between the breakfast types ( $\mathrm{Z}$-statistic $=-0.057 ; p=0.954)$.

\section{Effects of Breakfast Type on Subsequent Dietary Behaviour:}

Energy, Nutrient and Food Groups Servings Intake:

Participants' post-breakfast energy, macronutrient, water, sodium and food group servings intakes over the $4 \mathrm{~h}$ period are summarised in Table 3. Individual energy intakes for all participants on the CM and FS trials are presented in Figure 2. Participants' post-breakfast intake of energy, fat and carbohydrate were not significantly different between CM and FS trials ( $p$ 's $>0.05)$. Participants' voluntarily consumed significantly more protein $(p=0.033)$ and sodium $(p=0.049)$ on the FS compared to CM trials. Neither the total mass of food and fluid consumed nor water intake differed 
significantly between trials $(p$ 's $>0.05)$. There was also no significant difference for participants' intake in the number of serves of the grains, refined grains, whole grains, fruit, dairy, fat equivalents and added sugars food groups categories $\left(p^{\prime} s>0.05\right)$. However, participants consumed significantly more servings of the protein foods $(p=0.035)$ and oil equivalents $(p=0.046)$ food group categories on the FS compared to CM trials.

\section{Meal Latency:}

Meal latency was not significantly different between CM and FS trials [180(100) vs 146(97) min; $\mathrm{Z}=-1.526, p=0.127 ; r=0.22]$.

\section{Subjective Appetite Ratings:}

Subjective ratings of hunger, desire to eat, fullness and thirst for FS and CM trials are presented in Figure 3. A series of 2 (Treatment) $\times 8$ (Time) analyses for subjective ratings revealed no significant main effect of the test-breakfast (Treatment) on hunger, desire to eat, fullness and thirst, $\mathrm{F}(1,24)=1.43, p=0.243 ; \mathrm{F}(1,24)=2.08, p=0.163 ; \mathrm{F}(1,24)=0.134, p=0.718 ; \mathrm{F}(1,24)=2.83$, $p=0.106$, respectively. A significant main effect of time on ratings of hunger, desire to eat, fullness and thirst was observed, $\mathrm{F}(2.7,63.9)=25.28, p<0.001 ; \mathrm{F}(3.1,74.5)=19.44, p<0.001 ; \mathrm{F}(2.8$, $66.9)=36.54, p<0.001 ; \mathrm{F}(2.7,65.1)=15.94, p<0.001$, respectively; such that the consumption of a test-breakfast (irrespective of type) reduced hunger, desire to eat and thirst, and increased fullness. No significant Treatment $\times$ Time interactions were observed for ratings of hunger: $F(3.4$, $80.9)=1.05, p=0.399$; desire to eat: $\mathrm{F}(3.0,72.9)=1.05, p=0.375$; fullness: $\mathrm{F}(3.5,84.1)=1.46$, $p=0.224$; and thirst: $\mathrm{F}(3.4,82.3)=0.88, p=0.468$. 


\section{Repeatability:}

Average meal latency, energy, protein, fat, carbohydrate, sodium, total mass and water intake during the familiarisation (trial 1) and corresponding repeated trial were compared to establish normal day-to-day variability in results. The CV for each parameter is presented in Table 4. Meal latency and total mass had the lowest CVs $(<25 \%)$, whereas fat and sodium intakes had the highest CVs $(>50 \%)$. The CV was also calculated for subjective ratings at each time point following breakfast consumption and are presented as an overall average in Table 4 . The CV for subjective ratings of palatability was calculated for the single measure post-breakfast (Table 4). Average CVs were lowest for ratings of fullness and palatability $(<13 \%)$ and greatest for hunger, desire to eat and thirst $(>20 \%)$.

\section{Trial Order Analysis:}

Analysis of selected data based on the order of trial completion indicated no significant effects of trial order for either meal latency $\left(\mathrm{X}^{2}(2)=0.442, p=0.802\right)$ or energy intake $\left(\mathrm{X}^{2}(2)=0.207\right.$, $p=0.902$ ); indicating that participants did not alter their dietary behaviour as a result of 'exposure' to the study environment.

\section{Discussion:}

This laboratory study compared the effect of consuming CM and a nutritionally similar FS for breakfast on subsequent acute $(4 \mathrm{~h})$ dietary intake. Overall, results indicated no differences in subsequent intake of energy, macronutrients (except protein), food groups servings (except protein foods and oil equivalents), subjective ratings of hunger, and meal latency (i.e. time from breakfast completion to consuming the next calorie-containing food/beverage) between the two breakfasts. 
Furthermore, data for the repeated trials (i.e. FS or CM consumed on trial 1 vs equivalent repeated condition on trial 2 or 3 ) highlighted that dietary behaviour of participants was highly variable (e.g. $\mathrm{CV}$ for $\mathrm{EI}=41 \pm 43 \%$ ). Thus, the consumption of a nutritionally comparable breakfast either as CM or FS does not appear to modulate subsequent acute dietary intake.

In the $4 \mathrm{~h}$ following consumption of a FS, participants consumed $\sim 22 \%$ more energy compared to the CM breakfast trial. This outcome was not statistically significant, and well below the variability observed in our repeated trial $(\sim 41 \%)$. The post-prandial energy intake data is in agreement with findings from our previous study, which was similar in nature, but conducted in a free-living setting (McCartney et al., 2019) and also recent work of Rogers and Shahrokni (2018). Furthermore, while Houchins and colleagues (2013) identified an increase in energy intake following the consumption of a liquid vs solid fruit preload, this was only evident in participants classified as overweight/obese. Collectively, these studies suggest that when matched for nutrients, the food-form of breakfast (solid vs semi-solid vs liquid) only has a subtle influence on subsequent energy intake, and the change is well within typical "day-to-day" variation. In contrast, when a FS contains additional energy, this is unlikely to be off-set by a subsequent reduction in ad libitum energy intake (McCartney et al., 2019), making attempts to manage excess body weight more challenging.

Contrary to our hypothesis, results of the present study indicate that meal latency following consumption of breakfast was similar, irrespective of the breakfast consumed. This finding is inkeeping with results observed by Houchins and colleagues (2013), who found no difference in subsequent meal latency after consuming either a solid or liquid preload ( $290 \pm 24 \mathrm{vs} 271 \pm 22 \mathrm{~min}$, respectively). Collectively, these results suggest that the food-form (solid vs semi-solid vs liquid) of preloads do not alter food seeking behaviours. However, meal latency was overtly measured in 
the study by Houchins and colleagues (2013); participants were asked to document their subsequent dietary behaviours after leaving the laboratory, which increases the potential for participants to alter their normal eating behaviours (Robinson et al., 2014). Participants in the current study were covertly monitored via administration of a sham cognitive task, with participants only commencing the task (and the time being documented) on first consumption of calorie-containing foods/beverages. However, the sensitivity of this approach is unknown, as participants may have consumed food/energy-containing beverages prior to completing the task. Other methods of covertly monitoring meal latency may provide greater insight (with more sensitivity) into subsequent dietary behaviours following administration of a preload meal. For example, future research could employ methods such as video recording participants or isolating them to a room that allows behaviours to be observed without their knowledge (e.g. rooms equipped with one-way mirrors/windows).

Compared to non-viscous drinks, consumption of viscous beverages (e.g. smoothies) increases oral exposure time and/or may influence gastric emptying, which may result in beverage consumption facilitating greater exposure to sensory signals (similar to semi-solid and solid foods) involved in feelings of satiety (Krop et al., 2018). While a recent study has indicated that the consumption of iso-caloric beverages with different viscosities (i.e. blackcurrant squash, milk and a FS) do not influence subsequent energy intake, these beverages differ in many organoleptic and subjective properties (e.g. sweetness, bitterness, palatability etc.) (Rogers and Shahrokni, 2018). Further research is required to determine if smoothie characteristics (such as viscosity) influence subsequent dietary behaviours and intakes.

Results from the present study indicate that both CM and FS breakfasts induce comparable feelings of hunger, desire to eat, thirst and fullness. Previous laboratory research has suggested 
that the consumption of solid food items induce greater post-prandial increases in fullness and reduced hunger (Rogers and Shahrokni, 2018, Houchins et al., 2013). However, as discussed, these apparently beneficial responses do not always translate to changes in subsequent eating behaviour. Comparing subjective appetite responses between studies is challenging, as responses are likely to be influenced by many factors including individual participant characteristics (e.g. usual meal timings and volumes) and related methodological variables (e.g. relative size of the standardised meal, and timing of the post-prandial subjective measures). For example, the appetite responses to the drinks provided in the study by Rogers and Shahrokni (2018) were similar when participants were asked $\sim 2$ min after the different pre-load conditions. In contrast, when the same questions were asked to participants required to undertake a $2 \mathrm{~h}$ delay prior to the test meal intake, the milk and FS beverages induced greater feelings of fullness, than reported on the water and fruit juice trials. While it is clear that feelings of satiety are a function of time (Blundell et al., 2010), the results of Rogers and Shahrokni (2018) highlight that subjective appetite responses represent a complex interaction between the food/fluid provided, individual characteristics and methodological variables.

One unique component of the present study was the exploration of normal day-to-day variability between repeated trials. To our knowledge, no previous preload study has employed a reliability component as part of the study protocol to determine if subsequent eating behaviours are consistent. The present study calculated CV's of $\sim 21 \%$ for meal latency, $\sim 41 \%$ for energy and between $\sim 24 \%$ to $53 \%$ for other nutritional outcomes (i.e. macronutrients and sodium). In addition, CV's for subjective appetite ratings ranged from $\sim 11$ to $30 \%$. These findings indicate that individuals' acute eating behaviours are highly variable, even when the laboratory conditions are standardised and prior eating patterns (i.e. size and frequency of usual breakfast) are considered. 
Eating behaviours can be influenced by a range of factors such as dietary components (e.g. food palatability), cultural and social pressures, cognitive factors (e.g. stress, health attitudes, anxiety and depression) and genetic and epigenetic influences on personality characteristics (Leng et al., 2017). In addition, physical activity levels (King, 1998), hormonal fluctuations (Coll et al., 2007) and sleep duration (Cappuccio et al., 2008) can affect food choices and dietary behaviours. While these factors can be monitored, they may be difficult to control. It is possible that a combination of these factors contributed to the variability in eating behaviours observed in the current study.

\section{Conclusion:}

Results from the current study suggest that consumption of a nutrient-matched FS for breakfast does not influence acute dietary intake. While smoothies are a versatile medium that may facilitate the promotion of fruit and vegetables, the long-term effect of consuming FS in lieu of a cerealbased breakfast on outcomes such as body weight and health biomarkers remain unknown.

\section{Acknowledgements:}

We acknowledge the participants who volunteered to be a part of this research project.

\section{Disclosure Statement:}

All authors declare no potential conflict of interest.

\section{References:}

Academy of Nutrition and Dietetics. (2014). Adult Weight Management (AWM) guidelines. Retrieved from https://www.andeal.org/vault/pq132.pdf 
Almiron-Roig, E, Palla, L, Guest, K, Ricchiuti, C, Vint, N, Jebb, SA \& Drewnowski, A. 2013. Factors that determine energy compensation: a systematic review of preload studies. Nutr Rev. 71(7): 458-473.

Armstrong, LE, Pumerantz, AC, Fiala, KA, Roti, MW, Kavouras, SA, Casa, DJ \& Maresh, CM. 2010. Human Hydration Indices: Acute and Longitudinal Reference Values. Int J Sport Nutr Exerc Metab. 20(2): 145-153.

Bates, D \& Price, J. 2015. Impact of Fruit Smoothies on Adolescent Fruit Consumption at School. Health Educ Behav. 42(4): 487-492.

Berti, C, Riso, P, Brusamolino, A \& Porrini, M. 2015. Benefits of breakfast meals and pattern of consumption on satiety-related sensations in women. International Journal of Food Sciences and Nutrition. 66(7): 837-844.

Blundell, J, De Graaf, C, Hulshof, T, Jebb, S, Livingstone, B, Lluch, A, Mela, D, Salah, S, Schuring, E, Van Der Knaap, H \& Westerterp, M. 2010. Appetite control: methodological aspects of the evaluation of foods. Obes Rev. 11(3): 251-270.

British Dietetic Association. (2016). Healthy breakfast. Retrieved from https://www.bda.uk.com/foodfacts/healthy_breakfast

Cappuccio, FP, Taggart, FM, Kandala, N-B, Currie, A, Peile, E, Stranges, S \& Miller, MA. 2008. Meta-Analysis of Short Sleep Duration and Obesity in Children and Adults. Sleep. 31(5): 619626.

Coll, AP, Farooqi, IS \& O'Rahilly, S. 2007. The Hormonal Control of Food Intake. Cell. 129(2): 251-262. 
Constain, GA, Ricardo, CR, Rodríguez-Gázquez, LM, Alvarez, MG, Marín, CM \& Agudelo, CA. 2014. Diagnostic validity and usefulness of the Eating Attitudes Test-26 for the assessment of eating disorders risk in a Colombian female population. Atencion primaria. 46(6): 283-289.

Davis, CS, Clarke, RE, Coulter, SN, Rounsefell, KN, Walker, RE, Rauch, CE, Huggins, CE \& Ryan, L. 2016. Intermittent energy restriction and weight loss: a systematic review. Eur J Clin Nutr. 70(3): 292-9.

de Lauzon, B, Romon, M, Deschamps, V, Lafay, L, Borys, J, Karlsson, J, Ducimetiere, P \& Charles, M. 2004. The Three-Factor Eating Questionnaire-R18 Is Able to Distinguish among Different Eating Patterns in a General Population. J Nutr. 134(9): 2372-2380.

Dehghan, M \& Merchant, AT. 2008. Is bioelectrical impedance accurate for use in large epidemiological studies? Nutr J. 7(26): 1-7.

Fallaize, R, Wilson, L, Gray, J, Morgan, LM \& Griffin, BA. 2013. Variation in the effects of three different breakfast meals on subjective satiety and subsequent intake of energy at lunch and evening meal. European Journal of Nutrition. 52(4): 1353-1359.

Flood-Obbagy, JE \& Rolls, BJ. 2009. The effect of fruit in different forms on energy intake and satiety at a meal. Appetite. 52(2): 416-422.

Food Safety Authority of Ireland. (2011). Scientific recommendations for healthy eating guidelines.

Retrieved

from https://www.fsai.ie/recommendationsforhealthyeatingguidelinesinireland.html

Haines, PS, Guilkey, DK \& Popkin, BM. 1996. Trends in breakfast consumption of US adults between 1965 and 1991. J Am Diet Assoc. 96(5): 464-70. 
Houchins, JA, Tan, SY, Campbell, WW \& Mattes, RD. 2013. Effects of fruit and vegetable, consumed in solid vs beverage forms, on acute and chronic appetitive responses in lean and obese adults. Int J Obes. 37(8): 1109-1115.

Kant, AK \& Graubard, BI. 2006. Secular trends in patterns of self-reported food consumption of adult Americans: NHANES 1971-1975 to NHANES 1999-2002. Am J Clin Nutr. 84(5): 121523.

King. 1998. The relationship between physical activity and food intake. Proceedings of the Nutrition Society. 57(1): 77-84.

Krop, EM, Hetherington, MM, Nekitsing, C, Miquel, S, Postelnicu, L \& Sarkar, A. 2018. Influence of oral processing on appetite and food intake - A systematic review and meta-analysis. Appetite. 125: 253-269.

Leng, G, Adan, RAH, Belot, M, Brunstrom, JM, de Graaf, K, Dickson, SL, Hare, T, Maier, S, Menzies, J, Preissl, H, Reisch, LA, Rogers, PJ \& Smeets, PAM. 2017. The determinants of food choice. Proceedings of the Nutrition Society. 76(3): 316-327.

Marsh-Richard, DM, Hatzis, ES, Mathias, CW, Venditti, N \& Dougherty, DM. 2009. Adaptive Visual Analog Scales (AVAS): a modifiable software program for the creation, administration, and scoring of visual analog scales. Behav Res Methods. 41(1): 99-106.

McCartney, D, Langston, K, Desbrow, B, Khalesi, S \& Irwin, C. 2019. The influence of a fruit smoothie or cereal and milk breakfast on subsequent dietary intake: a pilot study. Int J Food Sci Nutr. 70(5): 612-622.

McCartney, D, Rattray, M, Desbrow, B, Khalesi, S \& Irwin, C. 2018. Smoothies: Exploring the Attitudes, Beliefs and Behaviours of Consumers and Non-Consumers. Curr Res Nutr Food Sci J. 6: 425-436. 
Mourao, DM, Bressan, J, Campbell, WW \& Mattes, RD. 2007. Effects of food form on appetite and energy intake in lean and obese young adults. Int J Obes. 31(11): 1688-95.

National Health and Medical Research Council. (2013). Dietary guidelines for all Australians. Retrieved from https://daa.asn.au/resource/breakfast-cereals-and-body-weight-fact-sheet/

New Zealand Ministry of Health. (2012). Food and nutrition guidelines for healthy children and young people (aged 2-18 years): a background paper. Partially revised February 2015. Wellington: Ministry of Health. Retrieved from https://www.health.govt.nz/publication/foodandnutrition-guidelines-healthy-children-and-young-people-aged-2-18-years-backgroundpaper

Ortega, R, Perez-Rodrigo, C \& López-Sobaler, A. 2015. Dietary Assessment Methods: Dietary Records. Nutricion Hospitalaria. 31: 38-45.

Pallant, J 2011. SPSS survival manual. A step by step guide to data analysis using SPSS for Windows, Berkshire, England, McGraw Hill.

Presser, HB \& Ward, BW. 2011. Nonstandard work schedules over the life course: a first look. Month Labor Rev. 134(7): 3-16.

Public Health England. (2016). The Eatwell Guide: Helping you eat a healthy, balanced diet. Retrieved from London: https://assets.publishing.service.gov.uk/government/uploads/system/uploads/attachment_dat a/file/742750/Eatwell Guide booklet 2018v4.pdf

Robinson, E, Kersbergen, I, Brunstrom, JM \& Field, M. 2014. I'm watching you. Awareness that food consumption is being monitored is a demand characteristic in eating-behaviour experiments. Appetite. 83: 19-25. 
Rogers, PJ \& Shahrokni, R. 2018. A Comparison of the Satiety Effects of a Fruit Smoothie, Its Fresh Fruit Equivalent and Other Drinks. Nutrients. 10(431): 1-14.

Rosi, A, Martini, D, Scazzina, F, Dall'Aglio, E, Leonardi, R, Monti, L, Fasano, F, Di Dio, C, Riggio, L \& Brighenti, F. 2018. Nature and Cognitive Perception of 4 Different Breakfast Meals Influence Satiety-Related Sensations and Postprandial Metabolic Responses but Have Little Effect on Food Choices and Intake Later in the Day in a Randomized Crossover Trial in Healthy Men. The Journal of Nutrition. 148(10): 1536-1546.

Shirreffs, SM, Merson, SJ, Fraser, SM \& Archer, DT. 2004. The effects of fluid restriction on hydration status and subjective feelings in man. Br J Nutr. 91(6): 951-958.

Sievert, K, Hussain, SM, Page, MJ, Wang, Y, Hughes, HJ, Malek, M \& Cicuttini, FM. 2019. Effect of breakfast on weight and energy intake: systematic review and meta-analysis of randomised controlled trials. BMJ. 364(42): 1-12.

Spence, C. 2017. Breakfast: The most important meal of the day? Int J Gastr Food Sci. 8: 1-6.

The Neilson Company. (2014). From breakfast to 'breakfaster': Liquid breakfast buyers double over past 5 years. Retrieved from New York, USA https://www.nielsen.com/au/en/insights/article/2014/from-breakfast-to-breakfaster-liquidbreakfast-buyers-double-over-past-five-years/

van der Laan, LN, de Ridder, DTD, Viergever, MA \& Smeets, PAM. 2011. The first taste is always with the eyes: A meta-analysis on the neural correlates of processing visual food cues. NeuroImage. 55(1): 296-303.

Williams, P. 2005. Breakfast and the diets of Australian adults: an analysis of data from the 1995 National Nutrition Survey. Int J Food Sci Nutr. 56(1): 65-79. 
World Health Organization. 2018. Obesity and overweight [Online]. Geneva: World Health Organization. Available: https://www.who.int/news-room/fact-sheets/detail/obesity-andoverweight [Accessed].

Zizza, C, Siega-Riz, AM \& Popkin, BM. 2001. Significant increase in young adults' snacking between 1977-1978 and 1994-1996 represents a cause for concern! Prev Med. 32(4): 303-10. 


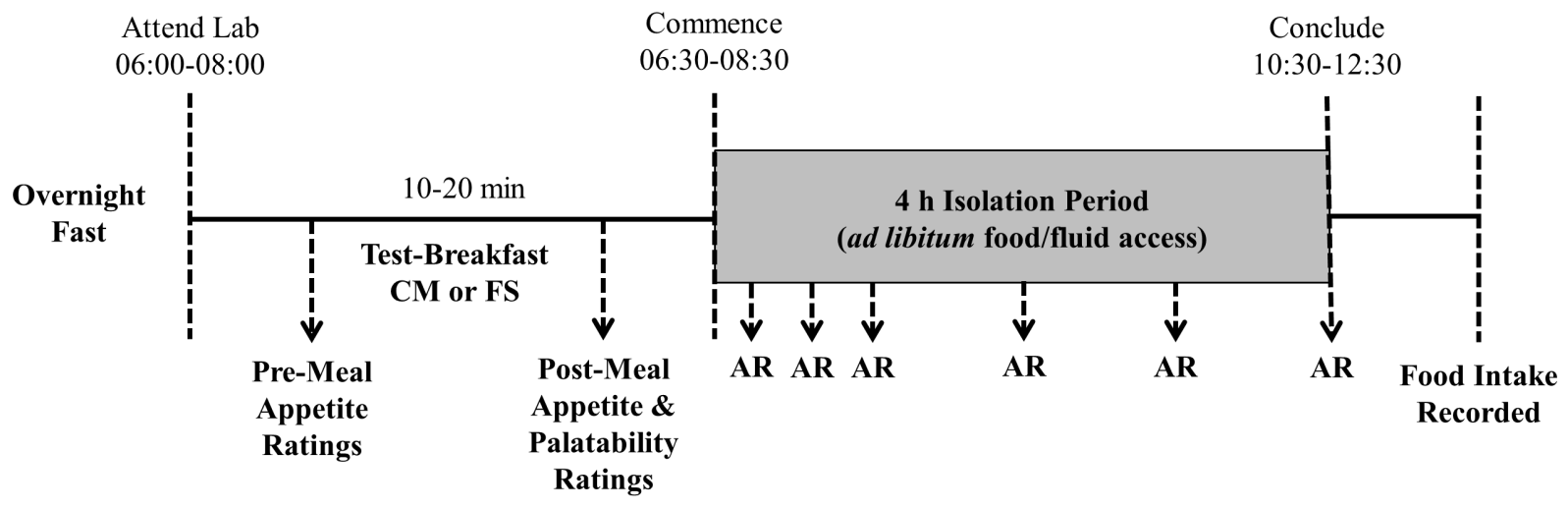

Figure 1. Schematic representation of experimental design employed; AR, appetite ratings; $(n=25)$. 


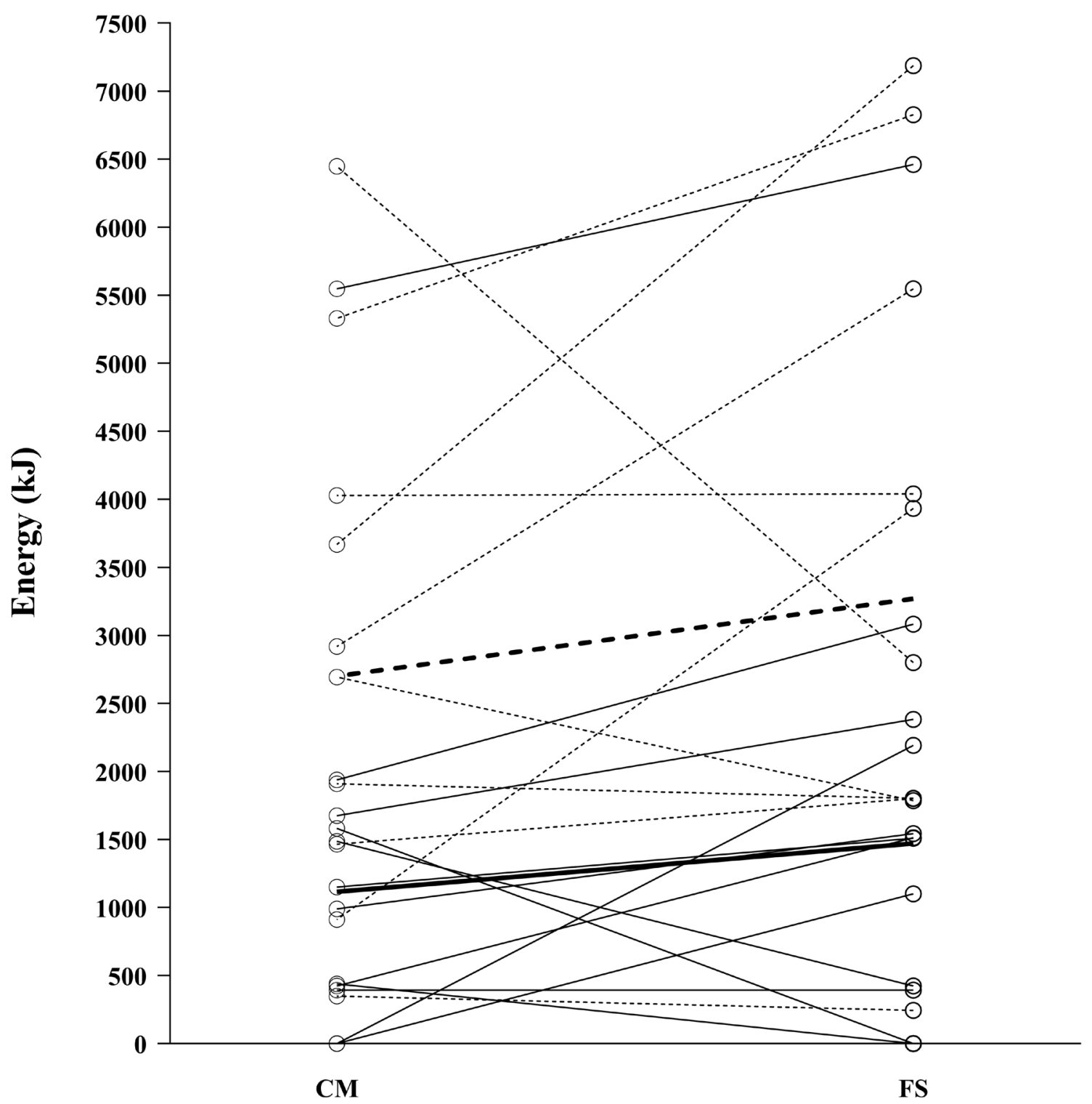

Trial

Figure 2. Post-breakfast energy consumption of individual participants during FS and CM trials $(n=25)$; Solid lines are females; Dashed lines are males; Thick lines represent the average energy consumption for females (dashed line) and males (solid line); kJ, kilojoules; CM, Cereal \& Milk; FS, Fruit Smoothie. 

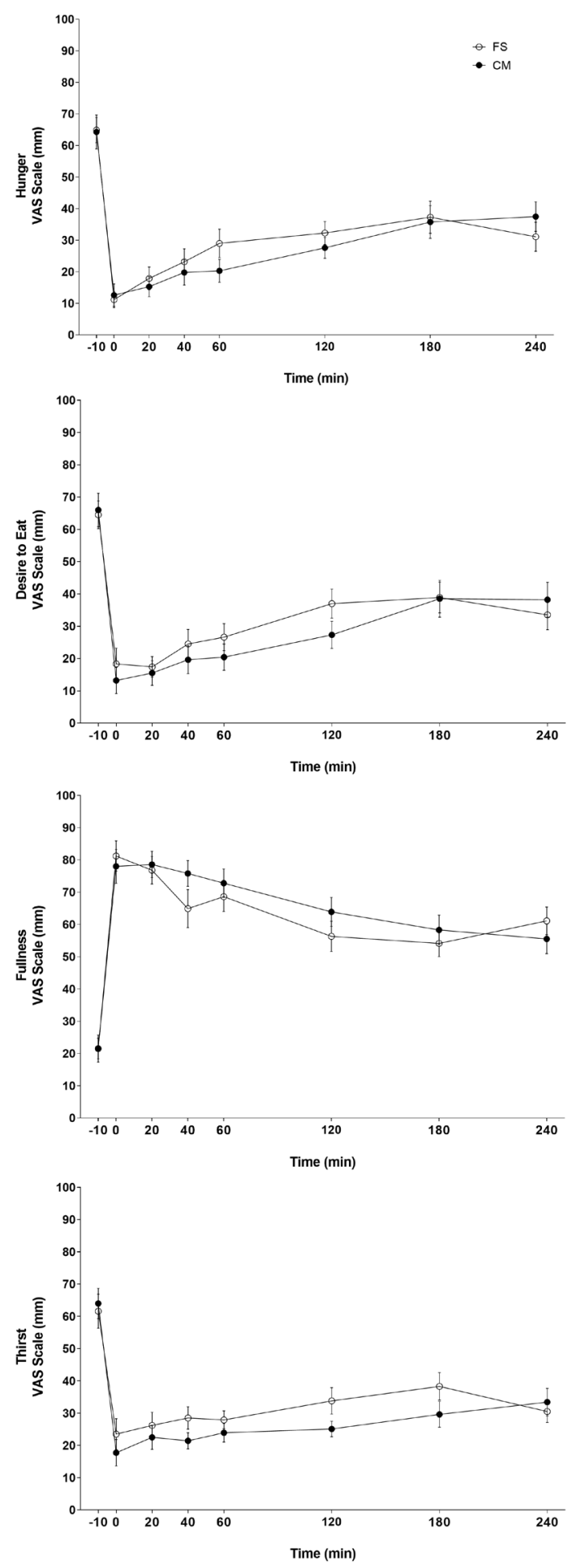

Figure 3. Subjective ratings of hunger, desire to eat, fullness and thirst $(n=25)$; CM, Cereal \& Milk $(\bullet)$; FS, Fruit Smoothie (०); values are Mean \pm SEM. 
Table 1. Food and beverages provided during the $4 \mathrm{~h}$ post-breakfast period

\begin{tabular}{lcc}
\hline Foods/Beverages (Brand) & Amount Available & Acceptability Rating \\
\hline Fruit and Nut Muesli Bar (Coles) & $3 \times 35 \mathrm{~g}$ bars & $8.5 \pm 2.6$ \\
Red Delicious Apples & $3 \times$ apples & $9.0 \pm 1.4$ \\
Cavendish Bananas & $3 \times$ bananas & $9.4 \pm 1.3$ \\
Sultanas (Sunbeam) & $150 \mathrm{~g}$ & $7.9 \pm 3.1$ \\
Raisin Toast (Tip Top) & $5 \times$ slices & $8.2 \pm 2.7$ \\
White/Multigrain Sandwich Bread (Coles) & $5 \times$ slices & $9.1 \pm 1.3$ \\
Single Sliced Tasty Cheese (Coon) & $1 \times 250 \mathrm{~g}$ packet & $8.4 \pm 2.3$ \\
Crunchy Peanut Butter (Bega) & $1 \times 375 \mathrm{~g}$ jar & $9.0 \pm 2.0$ \\
Honey (Woolworths) & $1 \times 375 \mathrm{~g}$ jar & $8.4 \pm 2.5$ \\
Strawberry Jam (IXL) & $1 \times 250 \mathrm{~g}$ jar & $8.0 \pm 2.8$ \\
Vegemite Yeast Spread (Bega) & $1 \times 150 \mathrm{~g}$ jar & $6.2 \pm 3.4$ \\
Margarine (Flora) & $1 \times 500 \mathrm{~g}$ container & $6.4 \pm 3.1$ \\
Vanilla Yoghurt (Yoplait) & $3 \times 175 \mathrm{~g}$ tubs & $8.0 \pm 3.2$ \\
Mini Chocolate Chip Cookies (Arnott's) & $3 \times 21 \mathrm{~g}$ packets & $8.6 \pm 2.9$ \\
Chocolate Brownies (Coles) & $3 \times 50 \mathrm{~g}$ brownies & $8.6 \pm 2.9$ \\
Plain Crinkle Cut Crisps (Smith's) & $3 \times 19 \mathrm{~g}$ packets & $8.0 \pm 3.0$ \\
Australian Almonds (Coles) & $150 \mathrm{~g}$ & $8.7 \pm 2.2$ \\
Natural Cashews (Coles) & $150 \mathrm{~g}$ & $8.6 \pm 2.2$ \\
Original Rice Cakes (Coles) & $150 \mathrm{~g}$ & $8.3 \pm 2.2$ \\
Banana Muffin Bars (Coles) & $3 \times 42 \mathrm{~g}$ bars & $8.0 \pm 3.0$ \\
Orange Juice (Coles) & $3 \times 600 \mathrm{ml} \mathrm{bottles}$ & $8.3 \pm 3.1$ \\
Water (Woolworths) & & - \\
\hline \hline
\end{tabular}

Acceptability Rating, Mean \pm SD rating of food items on a scale of 1 (dislike) to 10 (like). 
Table 2. Nutritional composition of participants' usual and experimental breakfasts $(n=25)$

\begin{tabular}{lccc}
\hline & FS & CM & Usual Breakfast \\
\hline Energy $(\mathrm{kJ})$ & $2006 \pm 701$ & $2006 \pm 701$ & $2112 \pm 892$ \\
Protein $(\mathrm{g})$ & $15.0 \pm 3.3$ & $15.6 \pm 3.4$ & $25.1 \pm 14.9$ \\
Carbohydrate $(\mathrm{g})$ & $73.4 \pm 32.6$ & $74.3 \pm 33.3$ & $50.2 \pm 24.4$ \\
Fibre $(\mathrm{g})$ & $10.0 \pm 2.2$ & $10.4 \pm 2.3$ & $7.3 \pm 4.5$ \\
Fat $(\mathrm{g})$ & $11.2 \pm 2.5$ & $10.8 \pm 2.4$ & $21.0 \pm 16.6$ \\
Water $(\mathrm{g})$ & $608 \pm 165$ & $607 \pm 133$ & $469 \pm 250$ \\
Total Mass $(\mathrm{g})$ & $691 \pm 165$ & $696 \pm 165$ & $578 \pm 266$ \\
Energy Density $\left(\mathrm{kJ} \cdot \mathrm{g}^{-1}\right)$ & $2.8 \pm 0.4$ & $2.8 \pm 0.4$ & $4.1 \pm 1.9$ \\
\hline \hline
\end{tabular}

CM, Cereal \& Milk; FS, Fruit Smoothie; Data are Mean \pm SD. 
Table 3. Energy, nutrient, food group servings intakes during the $4 \mathrm{~h}$ post-breakfast period $(n=25)$

\begin{tabular}{lccccc}
\hline & FS & CM & Z-statistic & $\boldsymbol{p}$-value & $\boldsymbol{r}$ \\
\hline Energy (kJ) & $1787(3190)$ & $1465(2436)$ & -1.651 & 0.099 & 0.23 \\
Protein (g) & $10(17)$ & $7(14)$ & -2.138 & $\mathbf{0 . 0 3 3}$ & 0.30 \\
Carbohydrate (g) & $63(89)$ & $40(73)$ & -0.991 & 0.322 & 0.14 \\
Fat (g) & $17(27)$ & $12(26)$ & -1.307 & 0.191 & 0.18 \\
Sodium (mg) & $229(604)$ & $175(485)$ & -1.972 & $\mathbf{0 . 0 4 9}$ & 0.28 \\
Water (g) & $635(589)$ & $630(512)$ & -0.730 & 0.465 & 0.10 \\
Total Mass (g) & $748(683)$ & $702(648)$ & -1.186 & 0.236 & 0.17 \\
Grains (\# serves) & $0.68(1.83)$ & $0.56(1.79)$ & -0.686 & 0.492 & 0.09 \\
Refined Grains (\# serves) & $0.00(1.21)$ & $0.00(0.99)$ & -0.966 & 0.334 & 0.14 \\
Whole Grains (\# serves) & $0.00(0.40)$ & $0.00(0.98)$ & -0.946 & 0.344 & 0.13 \\
Fruit (\# serves) & $0.70(2.03)$ & $1.00(2.03)$ & -1.008 & 0.313 & 0.14 \\
Protein Foods (\# serves) & $0.10(0.70)$ & $0.00(0.27)$ & -2.103 & $\mathbf{0 . 0 3 5}$ & 0.30 \\
Dairy (\# serves) & $0.00(0.31)$ & $0.00(1.02)$ & -0.868 & 0.386 & 0.12 \\
Oil Equivalents (\# serves) & $1.40(4.75)$ & $1.20(2.65)$ & -1.993 & $\mathbf{0 . 0 4 6}$ & 0.28 \\
Fat Equivalents (\# serves) & $0.30(2.80)$ & $0.20(2.85)$ & -1.080 & 0.280 & 0.15 \\
Added Sugars (\# serves) & $0.00(4.25)$ & $1.30(4.80)$ & -0.315 & 0.753 & 0.04 \\
\hline \hline
\end{tabular}

CM, Cereal \& Milk; FS, Fruit Smoothie; Data are Median (IQR); $r$, effect estimate calculated as the $\mathrm{Z}$ statistic divided by square root of $n$ (where $n=$ total number of observations). 
Table 4. Reliability between Familiarisation and Repeat trials $(n=25)$

\begin{tabular}{ll}
\hline Outcome & CV (\%) \\
\hline Energy (kJ) & $41 \pm 43$ \\
Protein (g) & $48 \pm 48$ \\
Fat (g) & $53 \pm 54$ \\
Carbohydrate (g) & $40 \pm 44$ \\
Sodium (mg) & $51 \pm 59$ \\
Total Mass (g) & $24 \pm 32$ \\
Total Water (g) & $28 \pm 37$ \\
Meal Latency (min) & $21 \pm 29$ \\
Hunger $(0-100)$ & $27 \pm 27$ \\
Thirst (0 - 100) & $21 \pm 19$ \\
Desire to Eat (0 - 100) & $30 \pm 24$ \\
Fullness (0 - 100) & $11 \pm 11$ \\
Palatability (0 - 100) & $12 \pm 23$ \\
\hline \hline
\end{tabular}

$\mathrm{CV}$, coefficient of variation; Data are Mean $\pm \mathrm{SD}$. 